\title{
Explanation for the mechanical strength of amyloid fibrils
}

\author{
Takeshi Fukuma, Anika S. Mostaert and Suzanne P. Jarvis* \\ Centre for Research on Adaptive Nanostructures and Nanodevices, Trinity College Dublin, Dublin 2, Ireland
}

Received 30 March 2006; accepted 2 June 2006; published online 6 July 2006

\begin{abstract}
The presence of "proteinaceous $\beta$-sheet rich fibrillar structures" and amyloidogenic material, has been alluded to extensively in the literature, in association with natural materials exhibiting superior mechanical strength per unit volume. Here we provide a clear experimental demonstration and explanation for why individual amyloid quaternary structures themselves have beneficial mechanical characteristics.
\end{abstract}

KEY WORDS: biotribology, nanotribology, adhesion, AFM

\section{Introduction}

Amyloid fibrils are quaternary protein structures formed from the non-specific folding (or misfolding), and subsequent aggregation, of proteins into intermolecular $\beta$-sheets of infinite propagation. These supramolecular crossed $\beta$-sheet structures are considered to be the fundamental motif of amyloid fibril architecture [1]. Whilst many aspects of the actual self-assembly process, and the internal structure of individual fibrils, remain unknown, evidence suggests that at least some of these aspects are amino-acid specific [1]. Amyloid fibrils are commonly associated with neurodegenerative diseases, although there is evidence that the amyloid structure is a generic form into which any polypeptide can fold, particularly in vitro under slightly denaturing conditions $[2,3]$. Recently there has been an interest in the mechanical properties of amyloid [4], which in part, has been triggered by the observed amyloidogenic nature of spider silk [5]. This amyloidogenic nature takes the form of a $\beta$-sheet enriching structural conversion of the major spider-silk protein, spidron, inside the silk gland of the spider. Similar conformational changes have been observed for the silkworm protein, fibroin, from random coil/helix to $\beta$-sheet [6]. This, together with the suspected nucleation-dependent aggregation mechanism during silk production [7], have lead to the suggestion that such conformational changes during the spinning process are as much (or more) important as (than) the protein sequence and native structure [6]. To date, direct measurements of the nanoscale surface mechanical properties of spider silk have not been able to provide additional insight, into the validity of this suggestion, or into the potential mechanical role of amyloid within the silk [8].

The amyloidogenic nature of natural adhesives and cements has also been described. In particular, Kamino

*E-mail: suzi.jarvis@tcd.ie et al. [9] observed a number of similarities between a barnacle underwater adhesive protein, and proteins involved in the formation of amyloid plaque. This, together with the insoluble behaviour of the adhesive protein, led them to speculate that the molecular mechanisms for forming an insoluble proteinaceous multimer may be similar between amyloid plaque and barnacle cement [9]. Recently, unusually well ordered, repetitive nanomechanical responses have been observed in the natural adhesive of terrestrial algae, with additional histochemical evidence suggesting that the mechanical responses can be attributed to the presence of amyloid within the material [10].

To date, the reason why quaternary amyloid structures may contribute to the exceptional mechanical strength of the materials described above, particularly under tension, has not been fully explored or explained.

Here we use an ultra-low noise atomic force microscope (AFM) [11] to investigate the tensile response of model amyloid fibrils formed in vitro (also known as amyloid-like fibrils [12]). In recent years, significant effort has been made to determine and understand the complex molecular structure of amyloid fibrils by investigating model amyloid fibrils such as these, formed from short peptide segments [13].

\section{Experimental Section}

\subsection{Materials}

Amyloid fibrils were formed from 11 amino acid long,

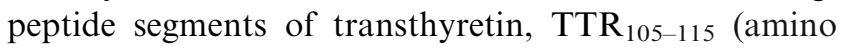
acid sequence Tyr-Thr-Ile-Ala-Ala-Leu-Leu-Ser-ProTyr-Ser) purchased from CS Bio Co., CA, U.S.A. The fibrils were formed following the method of Jaroniec et al. [14]. The TTR fibrils $(10 \mathrm{mg} / \mathrm{mL})$ were diluted $1: 5$ in MilliQ water. A $40 \mu \mathrm{L}$ sample was then deposited 
directly onto freshly cleaved mica, incubated for $60 \mathrm{~s}$, rinsed three times in MilliQ water, and dried in a stream of $\mathrm{N}_{2}$ gas. The deposited amyloid fibrils were first imaged and characterised at high resolution in air in order to confirm the distribution and density of fibrils on the mica surface, and to explore the structural morphology of the fibrils, figure 1 .

\subsection{AFM Measurements}

All measurements were performed with a custom designed, thermal noise limited AFM, capable of routine
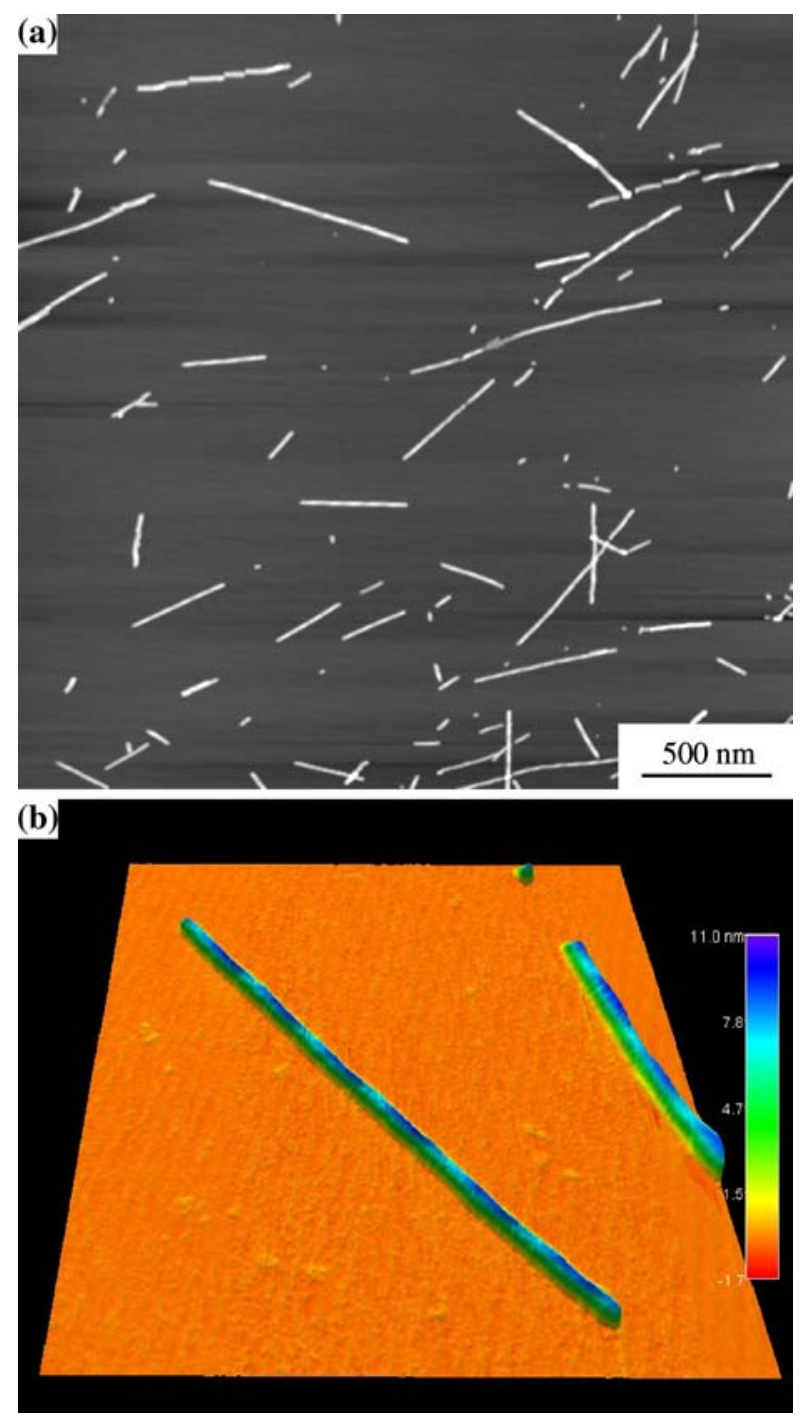

Figure 1. AFM images of TTR fibrils on mica in air obtained in intermittent-contact mode (cantilever spring constant, $k \approx 40 \mathrm{~N} /$ $\mathrm{m}$ and resonance frequency, $f_{0} \approx 300 \mathrm{kHz}$ ). Image sizes: (a) $3 \times 3 \mu \mathrm{m}^{2}$, (b) $600 \times 600 \mathrm{~nm}^{2}$. The length of the fibrils ranges from $100 \mathrm{~nm}$ to $2 \mu \mathrm{m}$ while the width is mostly within the range of $8 \pm 1 \mathrm{~nm}$. The height corrugation on top of the fibrils suggests that the fibrils consist of twisted protofilaments. The periodicity of the height corrugation measured along a fibril was about $90 \mathrm{~nm}$. true atomic resolution imaging on mica when operating in frequency-modulation detection mode [11]. The AFM is interfaced to a commercially available AFM controller (Asylum Research: MFP-3D Bipolar Controller).

High resolution imaging of fibrils was performed in air using intermittent-contact mode. For stability when imaging at high resolution, stiff silicon cantilevers (NCH: Nanosensors) with a spring constant of approx. $40 \mathrm{~N} / \mathrm{m}$ and resonance frequency of approx. $300 \mathrm{kHz}$ in air, were used.

The tensile response of the fibrils was measured in static pull off mode in MilliQ water to remove meniscus forces. In this case, compliant silicon cantilevers were used to optimise force sensitivity (BSI: Nanosensors), with spring constants in the range $0.05-0.07 \mathrm{~N} / \mathrm{m}$. The exact spring constant for each lever was calibrated using the method of Sader et al. [15]. For the pulling measurements, fibrils were located and imaged in intermittent-contact mode at the second resonance frequency of the cantilever (typically in the range $34-38 \mathrm{kHz}$ in water). Imaging resolution was found to degrade relative to imaging in air with stiffer levers, but was adequate for positioning the tip at specific locations along the length of the fibrils. Pulling measurements were made in static mode with tip velocities in the range of $20-30 \mathrm{~nm} / \mathrm{s}$.

\section{Results and Discussion}

Isolated fibrils were first imaged, and subsequently pulled (with non-specific binding to the tip) at different points along their length, in order to investigate their mechanical response. Various mechanical responses were observed with random force peaks and plateaus predominating. Irregularly spaced force peaks are typical of complex multi-molecular interactions. Figure 2 shows an example curve where a clear plateau was observed. Such plateaus often extended for tens of nanometres, which is far greater than either the width or the length of the individual peptide fragments. This response was attributed to the AFM tip attaching close to the end of an intermolecular $\beta$-sheet, as shown schematically to the right of figure 3. The sheet picked up by the tip is peeled from the sheet below at constant average force, resulting in a force plateau. This is because, with this pulling geometry, the bonds laterally connecting one $\beta$-sheet to the underlying sheet are loaded and broken in series. This mechanism was proposed and observed recently by Kellermayer et al. [16].

However, if the tip attaches to an intermolecular $\beta$-sheet somewhere along its length, then many of the bonds laterally connecting that sheet to the sheet below are loaded in parallel. This strongly pins the sheet to the bulk of the fibril and thus the applied tensile force can result in the successive unravelling of the peptide molecules from the bulk of the fibril, as shown schematically to 

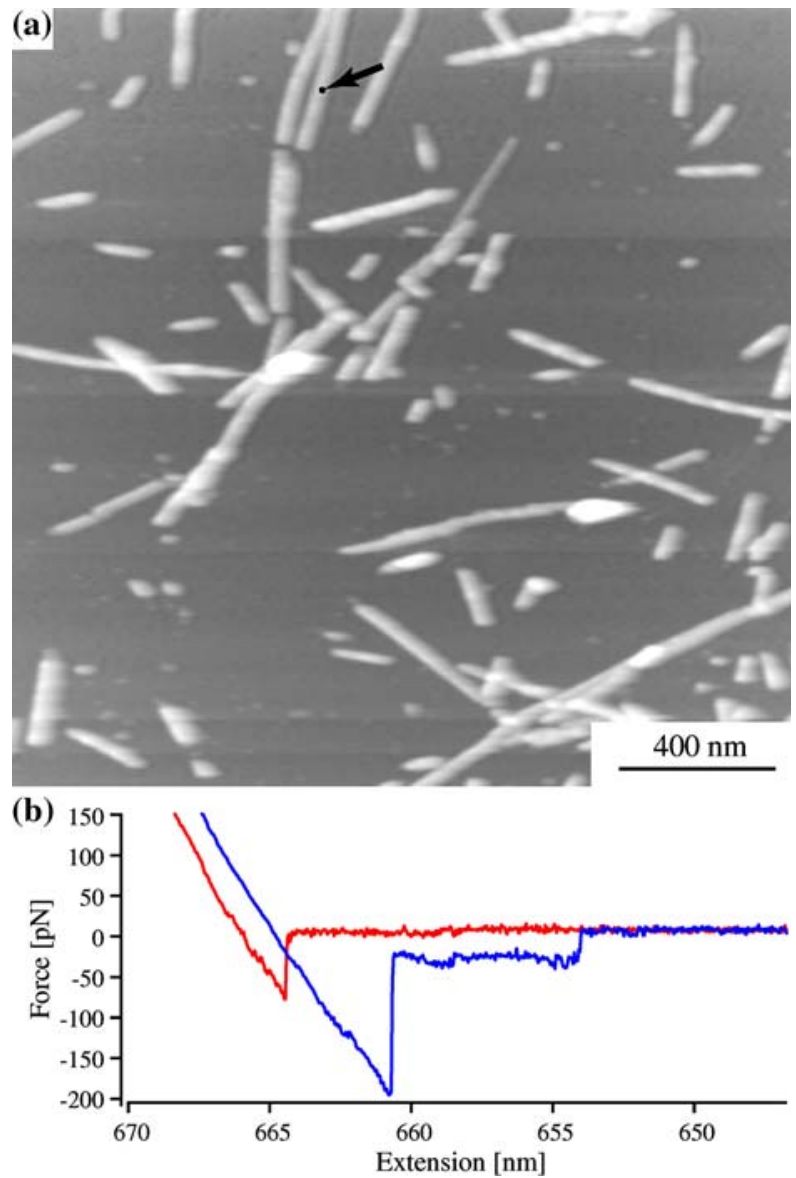

Figure 2. (a) AFM image $\left(2 \times 2 \mu \mathrm{m}^{2}\right)$ of TTR fibrils on mica in water obtained in intermittent-contact mode at the second resonance frequency of a cantilever $\left(k=0.057 \mathrm{~N} / \mathrm{m}\right.$ and $\left.f_{2}=34.9 \mathrm{kHz}\right)$. (b) Force versus extension curve measured on top of a fibril as indicated by a black arrow in (a). The measurement position was carefully chosen to be in the middle of the fibril. The red and blue lines correspond to the approaching and retracting curves, respectively. The tip velocity during the measurement was $20 \mathrm{~nm} / \mathrm{s}$. The measurement was performed in the static mode. The retraction force curve shows a plateau corresponding to a constant tensile force of $35 \mathrm{pN}$.

the left of figure 3 , and experimentally in figure 4 . The sawtooth mechanical response observed in the force versus extension curve of figure 4 relates to the sequential removal of the $\beta$-strands from within the intermolecular $\beta$-sheet. The magnitude of the force at the point the bonds rupture is approximately $20 \mathrm{pN}$ and will relate to both the number and type of bonds between the $\beta$-strands.

Despite our sawtooth mechanical response being associated with pulling on the bulk of the fibril it was observed less frequently than the plateau type response associated with pulling close to the end of the fibril. We found from imaging the fibrils after the measurement, that this was associated with the high probability of damaging the intermolecular $\beta$-sheets with the tip during the initial approach and indentation. This results in two 'ends' being created in the middle of the fibril, as seen in figure 5. We found that for these fibrils and AFM tips, compressive forces had to be kept to a minimum of around $100 \mathrm{pN}$ or less to avoid damaging the fibrils. The

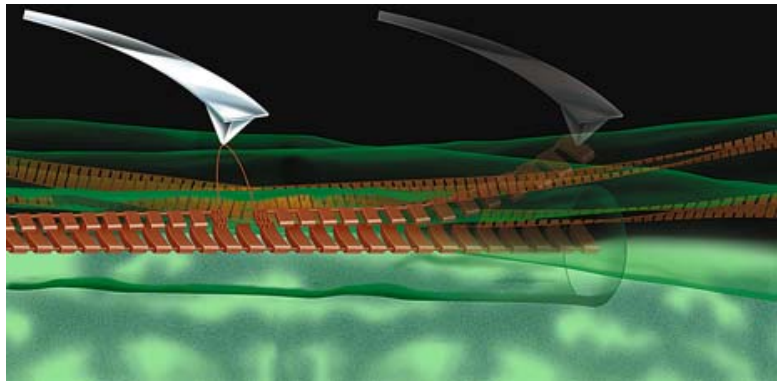

Figure 3. Schematic illustration of proposed mechanisms explaining, to the right, the peeling of an intermolecular $\beta$-sheet and, to the left, unravelling of the peptide molecules from the bulk of the fibril with an AFM tip. The schematic is not intended to suggest a specific molecular structure within the fibril. Interacting molecular building blocks in the form of $\beta$-strands run perpendicular to the direction of the fibril ( $\beta$-strand detail is shown only below the tip) and produce extended intermolecular $\beta$-sheets that form the fibril.
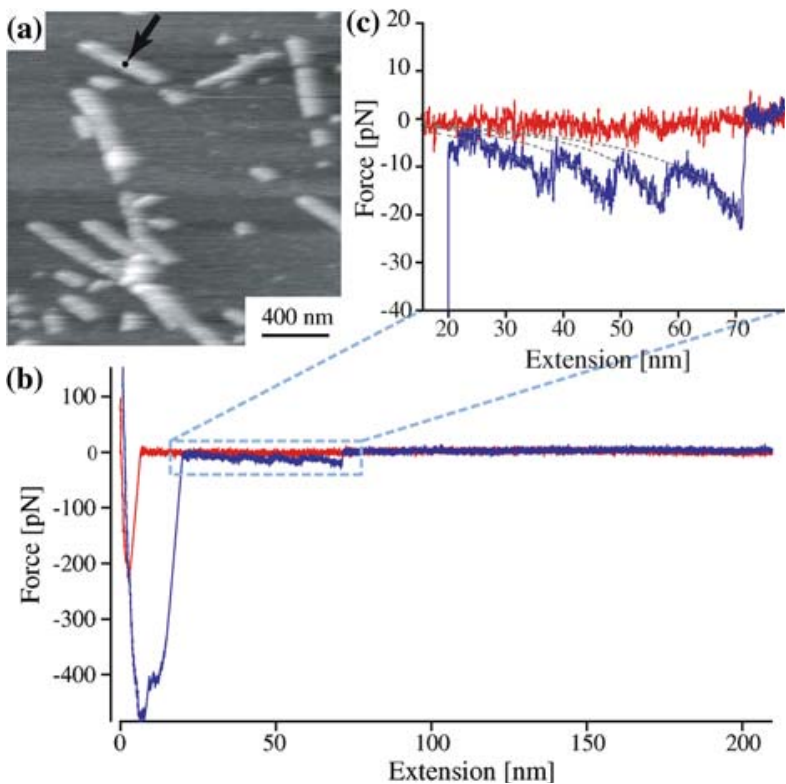

Figure 4. (a) AFM image $\left(2 \times 2 \mu \mathrm{m}^{2}\right)$ of TTR fibrils on mica in water obtained in intermittent-contact mode at the second resonance frequency of a cantilever $\left(k=0.066 \mathrm{~N} / \mathrm{m}\right.$ and $\left.f_{2}=37.7 \mathrm{kHz}\right)$. (b) Force versus extension curve measured on top of a fibril as indicated by a black arrow in (a). (c) An expansion of the force curve as indicated by blue dotted lines in (b). The force curve shows a sawtooth pattern, which was fitted with the worm-like-chain model as indicated by gray dotted lines. The curve shown in (b) is raw data while the one in (c) is averaged over five data points. The red and blue lines correspond to approaching and retracting curves, respectively. The tip velocity during the measurement was $30 \mathrm{~nm} / \mathrm{s}$.

cross-section along the length of the fibril in figure 5 shows a trough of approximately $60 \mathrm{~nm}$ in length and $1.3 \mathrm{~nm}$ in depth running along the fibril from the point of indentation. Intersheet distances are typically of the order of $1 \mathrm{~nm}$ [3], so it would seem likely that a single $\beta$-sheet has been peeled from the body of the fibril and has broken after peeling back $60 \mathrm{~nm}$. The data implies that the peeling mechanism pulls $\beta$-sheets from within the main fibril structure rather than removing loosely 

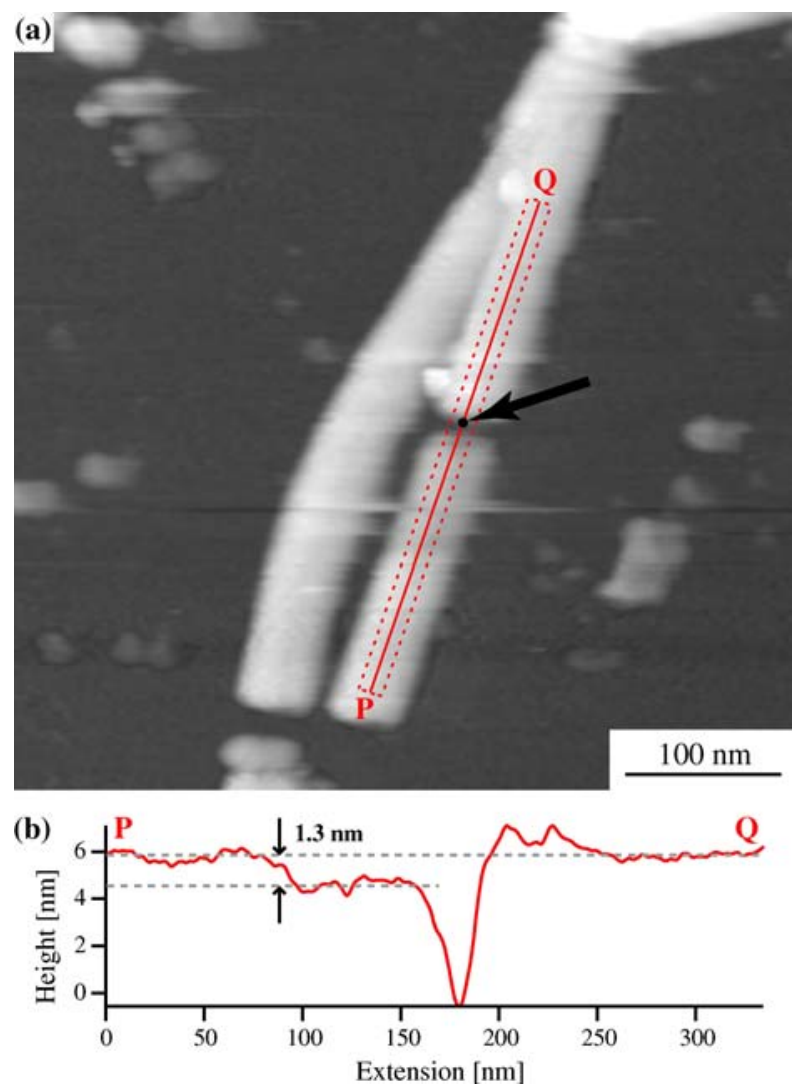

Figure 5. (a) AFM image $\left(500 \times 500 \mathrm{~nm}^{2}\right)$ of TTR fibrils on mica in water obtained in intermittent-contact mode at the second resonance frequency of a cantilever. The fibrils were imaged immediately after taking 42 force curves at the position indicated by a black arrow. One of the curves is shown in figure 2. (b) A cross-sectional profile measured along the line PQ shown in (a). The profile was averaged over 15 neighbouring pixels as indicated by a dotted line in (a). The profile reveals $1.3 \mathrm{~nm}$ height decrease of the fibril, suggesting the peeling of a $\beta$-sheet up to that point.

bound intermolecular $\beta$-sheets, which are not fully formed, and are lying at the surface of the fibril.

Previously Smith et al. [17] have explained how molecular level mechanical characteristics observed in adhesives, fibers and composites should contribute to their observed macroscopic mechanical strength. Specifically, they described how particular mechanical benefit is derived from a modular elongation mechanism [17]. This is regardless of the specific molecular composition and is due to the extra energy required to break the sacrificial bonds (typically within folded modular units or loops) before exposing the backbone of the material to a high enough force to induce rupture.

From the results shown in figure 4, this mechanism is clearly possible when a tensile load is applied at some point along the fibril. In a natural material, such as an adhesive, there should be a greater probability of tensile loading along the length of the fibril than at the ends, which are likely to be buried within the tangle of fibrils and other material in the adhesive matrix. It is also less likely that any compressive loading will damage the structure of the fibrils in natural materials for a number of reasons. Firstly the loading is unlikely to involve an object as sharp as an AFM tip. Secondly the fibrils are formed from whole proteins and are thus expected to be more robust (this could be further enhanced, by cross-linking between the proteins within the $\beta$-sheets). Finally the fibrils would commonly be in a soft biological matrix rather than lying directly on a rigid substrate.

We see qualitative similarities, between the mechanical responses of amyloids formed from peptide fragments and those formed from proteins in certain natural adhesives, measured previously [10]. Exact quantitative agreement is not observed, either in terms of the magnitude of the measured forces or the distance between the force peaks. This is probably because both parameters are likely to be highly dependent on the number of amino acids in the constituent proteins or polypeptide fragments. Each protein molecule or polypeptide fragment will form a $\beta$-strand or strands depending on their length. These molecules then self-assemble, to form intermolecular $\beta$-sheets, as shown schematically in figure 4. The peak force should increase if there are a greater number of amino acids in the constituent molecules because there are likely to be a higher number of "sacrificial bonds" and greater "hidden length". There is also a greater opportunity for cross-linking between whole proteins than there is for short peptides. Crosslinking will significantly enhance the continuity of the $\beta$-sheet and has already been shown to facilitate amyloid formation [18].

In terms of the mechanical properties of natural materials, the interaction of the fibrils with any adjacent biomaterial, polysaccharide for example, is also important. In other words, on the mesoscopic scale the fibrils would play a mechanical role by simply distributing any load, as in conventional fiber composite materials. At the nanoscale, any tensile force, regardless of the applied direction, should elicit the same beneficial mechanical characteristics of "hidden length" and "sacrificial bonds" from the fibril, due to the highly repetitive structure and high degree of axial symmetry. The fact that the fibrils can play a beneficial mechanical role both on the meso- and nano-scale explains why the quaternary structure is mechanically advantageous and may be the reason why the structure has been evolutionarily conserved in certain organisms. The fantastic ability of amyloid fibrils to self-assemble could also be mechanically advantageous as it implies that the fibrils may be able to rapidly self-heal under appropriate conditions.

\section{Conclusion}

We have provided a clear explanation for the apparent connection between the amyloid structure and natural materials of superior mechanical strength, based on the beneficial features of "hidden length" and 
"sacrificial bonds" present within the amyloid crossed$\beta$-sheet structure when under a tensile axial load. Given the generic nature of this amyloid crossed- $\beta$-sheet structure [2,3], it is feasible that this mechanism is the unidentified cause of superior mechanical strength in a broad range of natural materials.

Evidence suggests that the amyloid structure can form from any polypeptide, under appropriate conditions in vitro [3], making it a prime target for biomimicry. However, it is clear from our results that it will be necessary to explore methods of forming amyloid fibrils from cross-linked whole proteins, as opposed to short peptide segments, in order to reap the full benefit of these mechanical characteristics.

\section{Acknowledgments}

This work was funded by Science Foundation Ireland (01/PI.2/C033).

\section{References}

[1] J.D Sipe, Amyloid Proteins: The Beta Sheet Conformation and Disease (Wiley-VCH, Weinheim, Germany, 2005).

[2] C.E MacPhee and C.M. Dobson, J. Am. Chem. Soc. 122 (2000) 12707.
[3] M. Fändrich and C.M. Dobson, EMBO J. 21 (2002) 5682.

[4] S.H. Waterhouse and J.A. Gerrard, Aust. J. Chem. 57 (2004) 519.

[5] J.M. Kenney, D. Knight, M.J. Wise and F. Vollrath, Eur. J. Biochem. 269 (2002) 4159.

[6] L. Zhou, X. Chen, Z. Shao, P. Zhou, D.P. Knight and F. Vollrath, FEBS Lett. 554 (2003) 337.

[7] G.Y. Li, P. Zhou, Z.Z. Shao, X. Xie, X. Chen, H.H. Wang, L.J. Chunyu and T.Y. Yu, Eur. J. Biochem. 268 (2001) 6600.

[8] N. Becker, E. Oroudjev, S. Mutz, J.P. Cleveland, P.K. Hansma, C.Y. Hayashi, D.E. Makarov and H.G. Hansma, Nat. Mater. 2 (2003) 278.

[9] K. Kamino, K. Inoue, T. Maruyama, N. Takamatsu, S. Harayama and Y. Shizuri, J. Biol. Chem. 275 (2000) 27360.

[10] A.S. Mostaert, M.J. Higgins, T. Fukuma, F. Rindi and S.P. Jarvis, J. Biol. Phys. submitted.

[11] T. Fukuma and S.P. Jarvis, Rev. Sci. Inst. 77 (2006) 043701

[12] P. Westermark, M.D. Benson, J.N. Buxbaum, A.S. Cohen, B. Frangione, S. Ikeda, C.L. Masters, G. Merlini, M.J. Saraiva and J.D. Sipe, Amyloid 9 (2002) 197.

[13] E. Gazit, FEBS J. 272 (2005) 5971.

[14] C.P. Jaroniec, C.E. Mac Phee, N.S. Astrof, C.M. Dobson and R.G. Griffin, PNAS 99 (2002) 16748.

[15] J.E. Sader, J.W.M. Chon and P. Mulvaney, Rev. Sci. Instrum. 70 (1999) 3967.

[16] M.S.Z. Kellermayer, L. Grama, A. Karsai, A. Nagy, A. Kahn, Z.L. Datki and B. Penke, J. Biol. Chem. 280 (2005) 8464.

[17] B.L. Smith, T.E. Schäffer, M. Viani, J.B. Thompson, N.A. Frederick, J. Kindt, A. Belcher, G.D. Stucky, D.E. Morse and P.K. Hansma, Nature 399 (1999) 761.

[18] A.K. Das, M.G.B. Drew, D. Haldar and A. Banerjee, Org. Biomol. Chem. 3 (2005) 3502. 